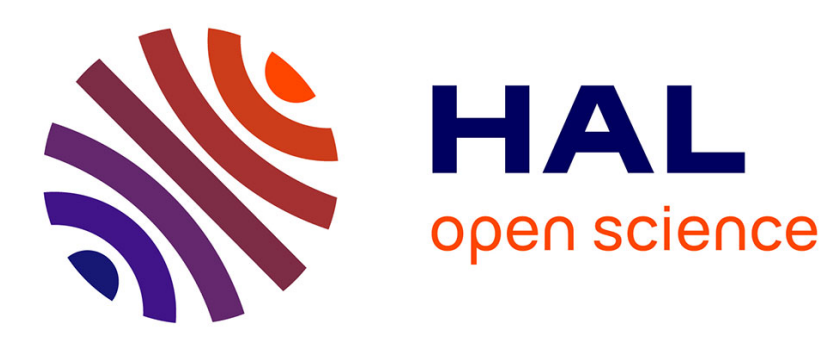

\title{
Quantitative Stability of a Generalized Equation
}

\author{
Samir Adly, Radek Cibulka
}

\section{To cite this version:}

Samir Adly, Radek Cibulka. Quantitative Stability of a Generalized Equation: Application to Nonregular Electrical Circuits. Journal of Optimization Theory and Applications, 2014, 160, pp.90-110. 10.1007/s10957-013-0369-6 . hal-00919288

\section{HAL Id: hal-00919288 \\ https://hal.science/hal-00919288}

Submitted on 18 Oct 2017

HAL is a multi-disciplinary open access archive for the deposit and dissemination of scientific research documents, whether they are published or not. The documents may come from teaching and research institutions in France or abroad, or from public or private research centers.
L'archive ouverte pluridisciplinaire HAL, est destinée au dépôt et à la diffusion de documents scientifiques de niveau recherche, publiés ou non, émanant des établissements d'enseignement et de recherche français ou étrangers, des laboratoires publics ou privés. 


\title{
Quantitative Stability of a Generalized Equation Application to Non-regular Electrical Circuits
}

\author{
S. Adly • R. Cibulka
}

\begin{abstract}
The paper is devoted to the study of several stability properties (such as Aubin/Lipschitz-like property, calmness and isolated calmness) of a special nonmonotone generalized equation. The theoretical results are applied in the theory of non-regular electrical circuits involving electronic devices like ideal diode, practical diode, and diode alternating current.
\end{abstract}

Keywords Generalized equation - Nonsmooth and variational analysis . Aubin/Lipschitz-like property · Calmness · Outer subdifferential ·

Non-regular circuits

\section{Introduction}

There are many particular applications in sciences and engineering where the model can be formulated in the form of the generalized equation. In this paper, we will focus on the models coming from electrical engineering. The complexity of modern integrated circuits makes the computer-aided circuit analysis inevitable in order to provide information about the behavior, performance and robustness of electrical circuits, by using accurate methods. Many software packages are used in the literature (in both industry and academic institutions) for the simulation of electronic circuits, among them we can cite, for example, SPICE, PLECS, PSIM, QUCS, etc.

S. Adly $(\varangle) \cdot$ R. Cibulka

XLIM UMR-CNRS 6172, Université de Limoges, 87060 Limoges, France

e-mail: samir.adly@unilim.fr 
In this paper, we study a class of non-regular circuits involving electrical devices like ideal diodes, practical diodes, Zener diodes, silicon controlled rectifiers or DIACs (Diodes for Alternating Current). It is well known, in the engineering community, that the study and the simulation of such nonsmooth electrical devices is not an easy task. Classical (smooth) mathematical and numerical analysis is applicable only to few problems. Therefore, natural extensions to the nonsmooth case are needed. Our methodology is based on tools from nonsmooth and convex analysis.

In [1], the authors considered the special case of the generalized equation (1) with the single-valued part being linear and the multivalued part being the Clarke subdifferential of the so-called Moreau-Panagiotopoulos super-potential. Our aim is to investigate the stability properties such as Aubin continuity, calmness and isolated calmness of the corresponding solution mapping. The verifiable conditions ensuring these properties are given in terms of the input data. The stability properties under parameter perturbations is a very important topic in engineering, not only for the determination of the behavior of a system with respect to perturbations but also for the construction of algorithms for the numerical simulation of the problem.

The originality of this work, compared to that in [1], lies in the fact that the singlevalued term may be nonlinear (which allows us sometimes to simplify the multivalued part of the generalized equation), and that the multivalued term is not necessarily a subdifferential. The main contribution of this paper is the study of the calmness property. More precisely, we give a sufficient condition ensuring the calmness property at a reference point; it is based on the outer subdifferential of a particular distance function (Proposition 5.1). This condition is not easy to check in practice in general. Nevertheless, we succeeded to apply this condition for a meaningful example from electrical engineering (Example 6.2). As far as we know, this is the first time that this kind of properties is applied to some concrete models in electronics.

The paper is organized as follows. In Sect. 2, we pose the problem and recall several standard notions from variational analysis. The three subsequent ones are devoted to the criteria ensuring the Aubin, the isolated calmness and the calmness properties of the solution mapping, respectively. Section 6 deals with some particular cases arising in electrical circuit theory. A conclusion is presented in Sect. 7.

\section{Preliminaries}

Given matrices $B \in \mathbb{R}^{n \times m}, C \in \mathbb{R}^{m \times n}$ with $m \leq n$, a vector $p \in \mathbb{R}^{n}$ and mappings $f: \mathbb{R}^{n} \rightarrow \mathbb{R}^{n}, F: \mathbb{R}^{m} \rightrightarrows \mathbb{R}^{m}$, we consider the problem of finding a solution $z \in \mathbb{R}^{n}$ to the generalized equation

$$
p \in f(z)+B F(C z) .
$$

We will refer to several additional assumptions in the main results. Namely,

(A1) $B$ is injective;

(A2) $f$ is continuously differentiable on $\mathbb{R}^{n}$;

(A3) $F$ has closed graph;

(A4) $C$ is surjective; and 
(A5) there are $F_{j}: \mathbb{R} \rightrightarrows \mathbb{R}, j \in\{1, \ldots, m\}$ such that $F(x)=\prod_{j=1}^{m} F_{j}\left(x_{j}\right)$ whenever $x=\left(x_{1}, \ldots, x_{m}\right)^{T} \in \mathbb{R}^{m}$.

In [1], the authors considered the special case of the above inclusion with the linear single-valued part $f$, with $B=C^{T}$, and $F$ being the Clarke subdifferential of the super-potential $J$ defined by

$$
J(x):=j_{1}\left(x_{1}\right)+j_{2}\left(x_{2}\right)+\cdots+j_{m}\left(x_{m}\right) \quad \text { whenever } x=\left(x_{1}, \ldots, x_{m}\right)^{T} \in \mathbb{R}^{m},
$$

with $j_{i}: \mathbb{R} \rightarrow \mathbb{R}$ being a locally Lipschitz continuous function for each index $i=$ $1, \ldots, m$.

The notation is fairly standard. In $\mathbb{R}^{d}$, the norm, the scalar product, the closed ball with the center $x \in \mathbb{R}^{d}$ with the radius $r \geq 0$, and the unit ball are denoted by $\|\cdot\|$, $\langle\cdot, \cdot\rangle, \mathbb{B}(x, r)$, and $\mathbb{B}$, respectively.

First, recall some well-known subdifferential concepts. Given a lower semicontinuous function $g: \mathbb{R}^{d} \rightarrow \overline{\mathbb{R}}:=\mathbb{R} \cup\{ \pm \infty\}$ and a point $x \in \mathbb{R}^{d}$ such that $g(x) \in \mathbb{R}$, the Fréchet/regular subdifferential of $g$ at $x$ is the set $\partial_{F} g(x)$ containing those $\xi \in \mathbb{R}^{d}$ that satisfy

$$
\liminf _{0 \neq h \rightarrow 0} \frac{g(x+h)-g(x)-\langle\xi, h\rangle}{\|h\|} \geq 0
$$

the limiting (Mordukhovich) subdifferential of $g$ at $x$ is the set $\partial g(x)$ containing all $\xi \in \mathbb{R}^{d}$ such that there are sequences $\left(x^{k}\right)_{k \in \mathbb{N}}$ and $\left(\xi^{k}\right)_{k \in \mathbb{N}}$ converging to $x$ and $\xi$, respectively, with

$$
g\left(x^{k}\right) \rightarrow g(x) \quad \text { as } k \rightarrow \infty, \quad \text { and } \quad \xi^{k} \in \partial_{F} g\left(x^{k}\right) \quad \text { for each } k \in \mathbb{N} \text {; }
$$

and finally, the outer subdifferential of $g$ at $x$ is the set $\partial_{>} g(x)$ containing those $\xi \in \mathbb{R}^{d}$ for which there are sequences $\left(x^{k}\right)_{k \in \mathbb{N}}$ and $\left(\xi^{k}\right)_{k \in \mathbb{N}}$ converging to $x$ and $\xi$, respectively, with

$$
g\left(x^{k}\right) \downarrow g(x) \quad \text { as } k \rightarrow \infty \quad \text { and } \quad \xi^{k} \in \partial_{F} g\left(x^{k}\right) \quad \text { for each } k \in \mathbb{N} .
$$

Note that nothing will change if for each $k \in \mathbb{N}$ one will take $\xi^{k}$ from $\partial g\left(x^{k}\right)$ instead of $\partial_{F} g\left(x^{k}\right)$ in the definition of the outer subdifferential. If $g(x)$ is infinite, then all the above subdifferentials of $g$ at $x$ are defined to be empty sets. Furthermore, note that the choice of any other (equivalent) norm on $\mathbb{R}^{d}$ (instead of the usual one induced by the scalar product) does not affect the above subdifferential constructions.

Given a non-empty subset $\Omega$ of $\mathbb{R}^{d}$, then $d(x, \Omega):=\inf \{\|y-x\|: y \in \Omega\}$ is the distance from $x \in \mathbb{R}^{d}$ to $\Omega$ and $\delta_{\Omega}$ denotes the indicator function of $\Omega$ (i.e., $\delta_{\Omega}(y)$ is equal to 0 if $y \in \Omega$ and to $+\infty$ otherwise). It is well known (e.g., see [2, p. 111]) that, if $\Omega$ is closed and does not contain $x \in \mathbb{R}^{d}$, then

$$
\partial d(\cdot, \Omega)(x)=\{(x-w) / d(x, \Omega): w \in \Omega \text { with }\|x-w\|=d(x, \Omega)\},
$$

and

$$
\partial_{F} d(\cdot, \Omega)(x)= \begin{cases}(x-w) / d(x, \Omega), & \text { if } \exists ! w \in \Omega \text { with } d(x, \Omega)=\|x-w\|, \\ \emptyset, & \text { otherwise. }\end{cases}
$$


Fix any $\bar{x} \in \Omega$ for a while; the Fréchet/regular normal cone to $\Omega$ at $\bar{x}$ is the set

$$
\widehat{N}(\bar{x} ; \Omega):=\partial_{F} \delta_{\Omega}(\bar{x})=\left\{\xi \in \mathbb{R}^{d}: \limsup _{\Omega \ni x \rightarrow \bar{x}} \frac{\langle\xi, x-\bar{x}\rangle}{\|x-\bar{x}\|} \leq 0\right\} ;
$$

similarly, $N(\bar{x} ; \Omega):=\partial \delta_{\Omega}(\bar{x})$ is the Mordukhovich limiting normal cone to $\Omega$ at $\bar{x}$ (which contains all $\xi \in \mathbb{R}^{d}$ for which there are sequences $\left(x^{k}\right)_{k \in \mathbb{N}}$ in $\Omega$ and $\left(\xi^{k}\right)_{k \in \mathbb{N}}$ in $\mathbb{R}^{d}$ converging to $\bar{x}$ and $\xi$, respectively, such that $\xi^{k} \in \widehat{N}\left(x^{k} ; \Omega\right)$ for each $\left.k \in \mathbb{N}\right)$; and, finally, the Bouligand-Severi tangent cone $T(\bar{x} ; \Omega)$ to $\Omega$ at $\bar{x}$ contains those $v \in \mathbb{R}^{d}$ for which there are sequences $\left(t^{k}\right)_{k \in \mathbb{N}}$ in $] 0, \infty\left[\right.$ and $\left(v^{k}\right)_{k \in \mathbb{N}}$ in $\mathbb{R}^{d}$ converging to 0 and $v$, respectively, such that $\bar{x}+t^{k} v^{k} \in \Omega$ whenever $k \in \mathbb{N}$.

By $S: \mathbb{R}^{d} \rightrightarrows \mathbb{R}^{l}$ we mean a multivalued mapping from $\mathbb{R}^{d}$ into $\mathbb{R}^{l}$ with the domain $\operatorname{dom} S$, the graph $\operatorname{gph} S$ and the range $\operatorname{rge} S$. Fix a point $(\bar{x}, \bar{y})$ in $\operatorname{gph} S$, then the contingent (graphical) derivative of $S$ at $(\bar{x}, \bar{y})$ is the mapping $D S(\bar{x}, \bar{y}): \mathbb{R}^{d} \rightrightarrows \mathbb{R}^{l}$ defined by

$$
D S(\bar{x}, \bar{y})(u):=\left\{v \in \mathbb{R}^{l}:(u, v) \in T((\bar{x}, \bar{y}) ; \operatorname{gph} S)\right\}, \quad u \in \mathbb{R}^{d} ;
$$

and the Mordukhovich coderivative of $S$ at $(\bar{x}, \bar{y})$ is the set-valued mapping $D^{*} S(\bar{x}, \bar{y}): \mathbb{R}^{l} \rightrightarrows \mathbb{R}^{d}$, assigning to each $\xi \in \mathbb{R}^{l}$ the set

$$
D^{*} S(\bar{x}, \bar{y})(\xi):=\left\{\eta \in \mathbb{R}^{d}:(\eta,-\xi) \in N((\bar{x}, \bar{y}) ; \operatorname{gph} S)\right\} .
$$

The mapping $S$ is said to have Aubin/Lipschitz-like property around $(\bar{x}, \bar{y})$ iff there is a constant $\kappa \geq 0$ along with neighborhoods $U$ of $\bar{x}$ and $V$ of $\bar{y}$ such that

$$
S(x) \cap V \subset S\left(x^{\prime}\right)+\kappa\left\|x-x^{\prime}\right\| \mathbb{B} \quad \text { for all } x, x^{\prime} \in U .
$$

The infimum of the set of values $\kappa$, for which there are neighborhoods $U$ of $\bar{x}$ and $V$ of $\bar{y}$ such that (4) holds, is called Lipschitz modulus of $S$ at $(\bar{x}, \bar{y})$ and is denoted by $\operatorname{lip}(S ;(\bar{x}, \bar{y}))$. It is well known (see [3, Theorem 3E.6]) that the Aubin/Lipschitz-like property of $S$ around $(\bar{x}, \bar{y})$ is equivalent to the metric regularity of $\Phi:=S^{-1}$ around $(\bar{y}, \bar{x})$ and that the regularity modulus of $\Phi$ at $(\bar{y}, \bar{x})$ equals the Lipschitz modulus of $S$ at $(\bar{x}, \bar{y})$.

Fixing one of the points in $U$, i.e. the request for the existence of a constant $\kappa \geq 0$ along with neighborhoods $U$ of $\bar{x}$ and $V$ of $\bar{y}$ such that

$$
S(x) \cap V \subset S(\bar{x})+\kappa\|x-\bar{x}\| \mathbb{B} \quad \text { whenever } x \in U,
$$

we get the definition of the calmness property of $S$ at $(\bar{x}, \bar{y})$. Again, the infimum of the set of values $\kappa$, for which there are neighborhoods $U$ of $\bar{x}$ and $V$ of $\bar{y}$ such that (5) holds, is called calmness modulus of $S$ at $(\bar{x}, \bar{y})$ and is denoted by $\operatorname{clm}(S ;(\bar{x}, \bar{y}))$. Remember that ref. [3, Theorem 3H.3] establishes the equivalence between calmness property of $S$ at $(\bar{x}, \bar{y})$ and the metric subregularity of $\Phi$ at $(\bar{y}, \bar{x})$. Moreover, the subregularity modulus of $\Phi$ at $(\bar{y}, \bar{x})$ and the calmness modulus of $S$ at $(\bar{x}, \bar{y})$ are the same.

Further, the mapping $S$ has the isolated calmness property at $(\bar{x}, \bar{y})$ provided that it has calmness property and $\bar{y}$ is an isolated point of $S(\bar{x})$, i.e. $S(\bar{x}) \cap \mathbb{B}(\bar{y}, r)=\{\bar{y}\}$ 
for some $r>0$. Note that the isolated calmness property of $S$ at $(\bar{x}, \bar{y})$ amounts to the existence of a constant $\kappa \geq 0$ along with neighborhoods $U$ of $\bar{x}$ and $V$ of $\bar{y}$ such that

$$
S(x) \cap V \subset \bar{y}+\kappa\|x-\bar{x}\| \mathbb{B} \quad \text { for each } x \in U .
$$

Theorem 3I.2 in [3] says that the isolated calmness property of $S$ at $(\bar{x}, \bar{y})$ is equivalent to the strong metric subregularity of $\Phi$ at $(\bar{y}, \bar{x})$.

From now on, we are going to use the following notations.

Standing Assumptions Denote by $\Phi$ the set-valued mapping from $\mathbb{R}^{n}$ into itself defined by $\Phi(z):=f(z)+B F(C z)$ whenever $z \in \mathbb{R}^{n}$. Set $S:=\Phi^{-1}$. Let us define the mappings $Q: \mathbb{R}^{n} \rightrightarrows \mathbb{R}^{n}$ and $F_{C}: \mathbb{R}^{m} \rightrightarrows \mathbb{R}^{m}$ by $Q(z):=B F(C z)$, $z \in \mathbb{R}^{n}$, and $F_{C}(u):=F(u)$ if $u=C z$ for some $z \in \mathbb{R}^{n}$ and $F_{C}(u):=\emptyset$ otherwise. We also suppose that we have in hand a point $(\bar{z}, \bar{p}) \in \operatorname{gph} \Phi$. Finally, put $\bar{v}:=\left(B^{T} B\right)^{-1} B^{T}(\bar{p}-f(\bar{z}))$.

\section{Aubin/Lipschitz-Like Property of $S$ at $(\bar{p}, \bar{z})$}

The following result provides the formula for the coderivative of the mapping $\Phi$ at the reference point.

Proposition 3.1 Under the assumptions (A1)-(A3), for any $\xi \in \mathbb{R}^{n}$ one has

$$
D^{*} \Phi(\bar{z}, \bar{p})(\xi)=\nabla f(\bar{z})^{T} \xi+C^{T} D^{*} F_{C}(C \bar{z}, \bar{v})\left(B^{T} \xi\right) .
$$

Proof Fix any $\xi \in \mathbb{R}^{n}$. As (A2) ensures the strict differentiability of $f$ at $\bar{z}$, ref. [2, Theorem 1.62(ii)] implies that

$$
D^{*} \Phi(\bar{z}, \bar{p})(\xi)=\nabla f(\bar{z})^{T} \xi+D^{*} Q(\bar{z}, \bar{p}-f(\bar{z}))(\xi) .
$$

Define $h: \mathbb{R}^{n} \times \mathbb{R}^{m} \rightarrow \mathbb{R}^{n}$ and $G: \mathbb{R}^{n} \rightrightarrows \mathbb{R}^{n}$ for each $z \in \mathbb{R}^{n}$ by $h(z, v):=B v, v \in$ $\mathbb{R}^{m}$, and $G(z):=F(C z)$. Thus $h(z, G(z))=\bigcup\{B v: v \in F(C z)\}=Q(z)$ whenever $z \in \mathbb{R}^{n}$. Moreover, $\bar{v} \in G(\bar{z})$ and $h(\bar{z}, \bar{v})=B \bar{v}=\bar{p}-f(\bar{z})$. Because of (A1), ref. [2, Lemma 1.126] in the finite-dimensional setting reveals that

$$
D^{*} Q(\bar{z}, \bar{p}-f(\bar{z}))(\xi)=D^{*} G(\bar{z}, \bar{v})\left(B^{T} \xi\right) .
$$

Finally, ref. [4, Theorem 3.10] yields that

$$
D^{*} G(\bar{z}, \bar{v})\left(B^{T} \xi\right)=C^{T} D^{*} F_{C}(C \bar{z}, \bar{v})\left(B^{T} \xi\right) .
$$

Combine the above expressions to conclude the proof.

Remark 3.1 This proposition is proved using the first order calculus rules instead of a second order chain rule used in [1], as it is valid for the particular case of $B=C^{T}$ only. 
The above statement allows us to characterize the Aubin/Lipschitz-like property of $S$ at the reference point.

Theorem 3.1 Under the assumptions (A1)-(A3), S has the Aubin/Lipschitz-like property at $(\bar{p}, \bar{z})$ if and only if

$$
0 \in \nabla f(\bar{z})^{T} \xi+C^{T} D^{*} F_{C}(C \bar{z}, \bar{v})\left(B^{T} \xi\right) \quad \Rightarrow \quad \xi=0 .
$$

Moreover, its Lipschitz modulus is given by

$$
\operatorname{lip}(S ;(\bar{p}, \bar{z}))=\sup \left\{\|\xi\|:\left(\nabla f(\bar{z})^{T} \xi+C^{T} D^{*} F_{C}(C \bar{z}, \bar{v})\left(B^{T} \xi\right)\right) \cap \mathbb{B} \neq \emptyset\right\} .
$$

Proof Note that $\eta \in D^{*} \Phi(\bar{z}, \bar{p})(\xi)$ if and only if $-\xi \in D^{*} S(\bar{p}, \bar{z})(-\eta)$. Having Proposition 3.1 in hand, one applies the well-known Mordukhovich criterion (see [2, Theorem 4.10]) in the finite-dimensional setting to conclude the proof.

Employing more assumptions, we may get the following corollaries.

Corollary 3.1 Suppose that the assumptions (A1)-(A4) hold true. Then S has the Aubin/Lipschitz-like property at $(\bar{p}, \bar{z})$ if and only if

$$
\left.\begin{array}{l}
\left(\left(C C^{T}\right)^{-1} C \nabla f(\bar{z})^{T} \xi, B^{T} \xi\right) \in-N((C \bar{z}, \bar{v}) ; \operatorname{gph} F) \\
\nabla f(\bar{z})^{T} \xi \in \operatorname{rge} C^{T}
\end{array}\right\} \Longrightarrow \quad \xi=0 .
$$

Proof Indeed, if $C$ is surjective, then $F_{C}=F$ and $C C^{T} \in \mathbb{R}^{m \times m}$ is non-singular.

First, let $\xi \in \mathbb{R}^{n}$ be such that $0 \in \nabla f(\bar{z})^{T} \xi+C^{T} D^{*} F(C \bar{z}, \bar{v})\left(B^{T} \xi\right)$. Find $w \in$ $D^{*} F(C \bar{z}, \bar{v})\left(B^{T} \xi\right)$ with $\nabla f(\bar{z})^{T} \xi+C^{T} w=0$. Thus $-\left(C C^{T}\right)^{-1} C \nabla f(\bar{z})^{T} \xi$ is in $D^{*} F(C \bar{z}, \bar{v})\left(B^{T} \xi\right)$. Clearly, we have $\nabla f(\bar{z})^{T} \xi \in \operatorname{rge} C^{T}$ and the definition of the coderivative of $F$ yields the rest.

On the other hand, pick any $\xi \in \mathbb{R}^{n}$ with $\left(\left(C C^{T}\right)^{-1} C \nabla f(\bar{z})^{T} \xi, B^{T} \xi\right)$ in $-N((C \bar{z}, \bar{v})$; gph $F)$ and $\nabla f(\bar{z})^{T} \xi \in \operatorname{rge} C^{T}$. The definition of the coderivative of $F$ says that $w:=-\left(C C^{T}\right)^{-1} C \nabla f(\bar{z})^{T} \xi \in D^{*} F(C \bar{z}, \bar{v})\left(B^{T} \xi\right)$. Thus $C C^{T} w=$ $-C \nabla f(\bar{z})^{T} \xi$. This implies that $C^{T} w+\nabla f(\bar{z})^{T} \xi \in \operatorname{ker} C \cap \operatorname{rge} C^{T}$. Therefore $0 \in \nabla f(\bar{z})^{T} \xi+C^{T} D^{*} F(C \bar{z}, \bar{v})\left(B^{T} \xi\right)$.

Remark 3.2 If (A5) also holds, then gph $F=\prod_{j=1}^{m}$ gph $F_{j}$, hence Proposition 6.41 in [5] implies that $N((C \bar{z}, \bar{v})$; gph $F)=\prod_{j=1}^{m} N\left(\left((C \bar{z})_{j}, \bar{v}_{j}\right)\right.$; gph $\left.F_{j}\right)$. Thus the first condition in (8) can be checked coordinate-wise.

Remark 3.3 In view of Theorem 1 in [6], the Aubin/Lipschitz-like property is stable with respect to a small perturbation of the function $f$. To be more precise, suppose that $S$ has the Aubin/Lipschitz-like property at $(\bar{p}, \bar{z})$. Then there exists $\varrho>0$ such that for every function $\tilde{f}: \mathbb{R}^{n} \rightarrow \mathbb{R}^{n}$, every point $(\tilde{p}, \tilde{z}) \in \mathbb{B}(\bar{p}, \varrho) \times \mathbb{B}(\bar{z}, \varrho)$ with

$$
\begin{aligned}
& \tilde{p} \in \tilde{f}(\tilde{z})+B F(C \tilde{z}), \quad\|\tilde{f}(\tilde{z})-f(\tilde{z})\| \leq \varrho, \quad \text { and } \\
& \left\|\left(\tilde{f}\left(z^{\prime}\right)-f\left(z^{\prime}\right)\right)-\left(\tilde{f}\left(z^{\prime \prime}\right)-f\left(z^{\prime \prime}\right)\right)\right\| \leq \varrho\left\|z^{\prime}-z^{\prime \prime}\right\| \quad \text { whenever } z^{\prime}, z^{\prime \prime} \in \mathbb{B}(\tilde{z}, \varrho),
\end{aligned}
$$

the mapping $\widetilde{S}:=(\tilde{f}+B F(C \cdot))^{-1}$ has the Aubin/Lipschitz-like property at $(\tilde{p}, \tilde{z})$. 


\section{Isolated Calmness of $S$ at $(\bar{p}, \bar{z})$}

In this case, we are going to compute the graphical derivative of $\Phi$ at the reference point. Let us start with the following geometric lemma which is only a slight generalization of Lemma 4.1 in [1] (where the first matrix in question was supposed to be surjective and $\Gamma$ was a closed set).

Lemma 4.1 Let $E \in \mathbb{R}^{k \times d}$ be any matrix, let $G \in \mathbb{R}^{l \times d}$ be injective, and let $\Gamma$ be a subset of $\operatorname{rge} E$. Put $\Xi:=E^{-1}(\Gamma)$ and $\Lambda:=G(\Xi)$. For $\bar{x} \in \Lambda$ denote by $\bar{y}$ the (unique) point in $\Xi$ with $G \bar{y}=\bar{x}$. Then

$$
T(\bar{x} ; \Lambda)=\left\{u \in \mathbb{R}^{l}: \exists w \in \mathbb{R}^{d} \text { such that } u=G w \text { and } E w \in T(E \bar{y} ; \Gamma)\right\} .
$$

Proof We claim that $T(\bar{y} ; \Xi)=\left\{w \in \mathbb{R}^{d}: E w \in T(E \bar{y}, \Gamma)\right\}$. First, take any $w \in$ $T(\bar{y} ; \Xi)$. Find $\left(t^{n}\right)_{n \in \mathbb{N}}$ in $] 0, \infty\left[\right.$ and $\left(w^{n}\right)_{n \in \mathbb{N}}$ in $\mathbb{R}^{d}$ converging to 0 and $w$, respectively, such that $\bar{y}+t^{n} w^{n} \in \Xi$ whenever $n \in \mathbb{N}$. Then we have that $E \bar{y}+t^{n} E w^{n}=$ $E\left(\bar{y}+t^{n} w^{n}\right) \in \Gamma$ for each $n \in \mathbb{N}$. Hence $E w \in T(E \bar{y} ; \Gamma)$. On the other hand, let $w \in \mathbb{R}^{d}$ be such that $E w \in T(E \bar{y}, \Gamma)$. Pick $\left(t^{n}\right)_{n \in \mathbb{N}}$ in $] 0, \infty\left[\right.$ and $\left(v^{n}\right)_{n \in \mathbb{N}}$ in $\mathbb{R}^{k}$ converging to 0 and $E w$, respectively, such that $E \bar{y}+t^{n} v^{n} \in \Gamma$ whenever $n \in \mathbb{N}$. As $\Gamma \subset \operatorname{rge} E$, where the latter set is a closed subspace of $\mathbb{R}^{k}$, one infers that $v^{n} \in \operatorname{rge} E$ for each $n \in \mathbb{N}$. Therefore, by Banach open mapping theorem there is $\left(w^{n}\right)_{n \in \mathbb{N}}$ in $\mathbb{R}^{d}$ converging to $w$ such that $E w^{n}=v^{n}$ for each $n \in \mathbb{N}$. Thus, for an arbitrary index $n$, we have $E\left(\bar{y}+t^{n} w^{n}\right) \in \Gamma$, hence $\bar{y}+t^{n} w^{n} \in E^{-1}(\Gamma)=\Xi$. So $w \in T(\bar{y} ; \Xi)$. The claim is proved.

Using exactly the same steps as in the proof of Lemma 4.1 in [1] one gets that $T(\bar{x} ; \Lambda)=\{G w: w \in T(\bar{y}, \Xi)\}$. This and the claim yield the assertion.

Proposition 4.1 Under the assumptions (A1)-(A2), for any $b \in \mathbb{R}^{n}$ one has

$$
D \Phi(\bar{z}, \bar{p})(b)=\nabla f(\bar{z}) b+B D F_{C}(C \bar{z}, \bar{v})(C b) .
$$

Proof Fix any $b \in \mathbb{R}^{n}$. By Proposition 4A.2 in [3], we have

$$
D \Phi(\bar{z}, \bar{p})(b)=\nabla f(\bar{z}) b+D Q(\bar{z}, \bar{p}-f(\bar{z}))(b) .
$$

Observe that

$$
\begin{aligned}
\operatorname{gph} Q=\left\{\left(\begin{array}{l}
u \\
v
\end{array}\right) \in \mathbb{R}^{2 n}: \exists\left(\begin{array}{l}
b \\
c
\end{array}\right) \in \mathbb{R}^{n} \times \mathbb{R}^{m}:\right. \\
\left.\left(\begin{array}{l}
u \\
v
\end{array}\right)=G\left(\begin{array}{l}
b \\
c
\end{array}\right) \text { and } E\left(\begin{array}{l}
b \\
c
\end{array}\right) \in \operatorname{gph} F_{C}\right\}
\end{aligned}
$$

with

$$
G:=\left(\begin{array}{cc}
I_{n} & 0 \\
0 & B
\end{array}\right) \quad \text { and } \quad E:=\left(\begin{array}{cc}
C & 0 \\
0 & I_{m}
\end{array}\right)
$$


As $B$ is injective, so is $G$. Lemma 4.1 (with $k:=2 m, l:=2 n, d:=n+m, \Gamma:=$ $\operatorname{gph} F_{C}, \bar{x}:=(\bar{z}, \bar{p}-f(\bar{z}))^{T}$, and $\left.\bar{y}:=(\bar{z}, \bar{v})^{T}\right)$ reveals that

$$
T((\bar{z}, \bar{p}-f(\bar{z})) ; \operatorname{gph} Q)=\left\{\left(\begin{array}{c}
b \\
B c
\end{array}\right):\left(\begin{array}{c}
C b \\
c
\end{array}\right) \in T\left((C \bar{z}, \bar{v}) ; \operatorname{gph} F_{C}\right)\right\} .
$$

This means that $D Q(\bar{z}, \bar{p}-f(\bar{z}))(b)=B D F_{C}(C \bar{z}, \bar{v})(C b)$. The assertion is proved.

Theorem 4.1 Under the assumptions (A1)-(A3), S has the isolated calmness property at $(\bar{p}, \bar{z})$ if and only if

$$
0 \in \nabla f(\bar{z}) b+B D F_{C}(C \bar{z}, \bar{v})(C b) \quad \Rightarrow \quad b=0 .
$$

Moreover, its calmness modulus is given by

$$
\operatorname{clm}(S ;(\bar{p}, \bar{z}))=\sup \left\{\|b\|:\left(\nabla f(\bar{z}) b+B D F_{C}(C \bar{z}, \bar{v})(C b)\right) \cap \mathbb{B} \neq \emptyset\right\} .
$$

Proof Note that $x \in D \Phi(\bar{z}, \bar{p})(y)$ if and only if $y \in D S(\bar{p}, \bar{z})(x)$. Combine Corollary 4C.2 and Theorem 4C.1 in [3] and Proposition 4.1 to conclude the proof.

Again, imposing additional assumptions we get the following statement.

Corollary 4.1 Suppose that the assumptions (A1)-(A4) hold true. Then $S$ has the isolated calmness property at $(\bar{p}, \bar{z})$ if and only if

$$
\left.\begin{array}{l}
\left(C b,-\left(B^{T} B\right)^{-1} B^{T} \nabla f(\bar{z}) b\right) \in T((C \bar{z}, \bar{v}) ; \operatorname{gph} F) \\
\nabla f(\bar{z}) b \in \operatorname{rge} B
\end{array}\right\} \Rightarrow b=0 .
$$

Proof Indeed, if $C$ is surjective, then $F_{C}=F$. Note that (A1) ensures that $B^{T} B \in$ $\mathbb{R}^{m \times m}$ is non-singular.

First, let $b \in \mathbb{R}^{n}$ be such that $0 \in \nabla f(\bar{z}) b+B D F(C \bar{z}, \bar{v})(C b)$. Find a point $w \in D F(C \bar{z}, \bar{v})(C b)$ with $\nabla f(\bar{z}) b+B w=0$. Thus $-\left(B^{T} B\right)^{-1} B^{T} \nabla f(\bar{z}) b$ is in $D F(C \bar{z}, \bar{v})(C b)$. Clearly, we have $\nabla f(\bar{z}) b \in \operatorname{rge} B$ and the definition of the contingent derivative of $F$ yields the rest.

On the other hand, pick any $b \in \mathbb{R}^{n}$ with $\left(C b,-\left(B^{T} B\right)^{-1} B^{T} \nabla f(\bar{z}) b\right)$ in $T((C \bar{z}, \bar{v})$; gph $F)$ and $\nabla f(\bar{z}) b \in \operatorname{rge} B$. The definition of the contingent derivative says that $w:=-\left(B^{T} B\right)^{-1} B^{T} \nabla f(\bar{z}) b \in D F(C \bar{z}, \bar{v})(C b)$. Thus we have $B^{T} B w=$ $-B^{T} \nabla f(\bar{z}) b$. So $B w+\nabla f(\bar{z}) b \in \operatorname{ker} B^{T} \cap \operatorname{rge} B=\{0\}$. Therefore $0 \in \nabla f(\bar{z}) b+$ $B D F(C \bar{z}, \bar{v})(C b)$.

Remark 4.1 If (A5) is also satisfied and gph $F_{j}$ is normally (Clarke) regular at $\left((C \bar{z})_{j}, \bar{v}_{j}\right)$ for each $j \in\{1, \ldots, m\}$ in sense of Definition 6.4 in [5], then Proposition 6.41 in [5] implies that $T((C \bar{z}, \bar{v}) ; \operatorname{gph} F)=\prod_{j=1}^{m} T\left(\left((C \bar{z})_{j}, \bar{v}_{j}\right) ; \operatorname{gph} F_{j}\right)$. Hence the first condition in (10) can be checked coordinate-wise.

In view of Theorem 3 in [6], also the isolated calmness property is stable with respect to the perturbation of $f$ in the sense discussed in Remark 3.3. 


\section{Calmness of $S$ at $(\bar{p}, \bar{z})$}

Let us point out that the criteria guaranteeing the calmness are more complicated than those for the isolated calmness. Hence, one should always consider the latter property first, and employ more sophisticated tools of this section only in case of their failure. One can derive the following sufficient condition for calmness.

Proposition 5.1 Suppose that the assumptions (A1)-(A3) are satisfied. Put $\Lambda=$ gph $F \times \operatorname{rge} B$ and define the functions $g: \mathbb{R}^{n} \rightarrow \mathbb{R}^{m} \times \mathbb{R}^{m} \times \mathbb{R}^{n}$ and $h: \mathbb{R}^{n} \rightarrow \mathbb{R}_{+}$ for each $z \in \mathbb{R}^{n}$ by $g(z):=\left(C z,\left(B^{T} B\right)^{-1} B^{T}(\bar{p}-f(z)), \bar{p}-f(z)\right)$ and $h(z):=$ $d(g(z), \Lambda)$. Then $S$ has the calmness property at $(\bar{p}, \bar{z})$, provided that

$$
0 \notin \partial_{>} h(\bar{z}) .
$$

Proof The inclusion (1) says that, for each $p \in \mathbb{R}^{n}$, one has

$$
S(p)=\left\{z \in \mathbb{R}^{n}:\left(C z,\left(B^{T} B\right)^{-1} B^{T}(p-f(z)), p-f(z)\right) \in \Lambda\right\} .
$$

Indeed, fix any $p \in \mathbb{R}^{n}$. First, pick any $z \in \mathbb{R}^{n}$ with $(z, p) \in \operatorname{gph} \Phi$. Inclusion (1) reveals that $p-f(z) \in \operatorname{rge} B$. We have already mentioned that $B^{T} B \in \mathbb{R}^{m \times m}$ is nonsingular. Hence, (1) implies that $\left(C z,\left(B^{T} B\right)^{-1} B^{T}(p-f(z))\right) \in \operatorname{gph} F$. On the other hand, fix an arbitrary $z \in \mathbb{R}^{n}$ with

$$
\left(C z,\left(B^{T} B\right)^{-1} B^{T}(p-f(z)), p-f(z)\right) \in \Lambda .
$$

Then $B^{T}(p-f(z))=B^{T} B w$ for some $w \in F(C z)$. As $p-f(z) \in \operatorname{rge} B$, we have $p-f(z)-B w \in \operatorname{ker} B^{T} \cap \operatorname{rge} B=\{0\}$. Therefore $(z, p) \in \operatorname{gph} \Phi$.

Define the mapping $M: \mathbb{R}^{m} \times \mathbb{R}^{m} \times \mathbb{R}^{n} \rightrightarrows \mathbb{R}^{n}$ as follows:

$$
M(y):=\left\{z \in \mathbb{R}^{n}: g(z)+y \in \Lambda\right\}, \quad y \in \mathbb{R}^{m} \times \mathbb{R}^{m} \times \mathbb{R}^{n} .
$$

As $\Lambda$ is closed and the mapping

$$
\mathbb{R}^{n} \times \mathbb{R}^{n} \ni(p, z) \mapsto\left(C z,\left(B^{T} B\right)^{-1} B^{T}(p-f(z)), p-f(z)\right) \in \mathbb{R}^{m} \times \mathbb{R}^{m} \times \mathbb{R}^{n}
$$

is continuously differentiable (hence strictly differentiable and therefore locally Lipschitz continuous), Lemma 1 in [7] says that $S$ is calm at $(\bar{p}, \bar{z})$, provided that so is $M$ at $(0, \bar{z})$. As observed in [7, p. 438], $M$ is calm at $(0, \bar{z})$ if and only if there are $L>0$ and $\varepsilon>0$ such that

$$
d\left(z, g^{-1}(\Lambda)\right) \leq L d(g(z), \Lambda) \quad \text { whenever } z \in \mathbb{B}(\bar{z}, \varepsilon) .
$$

Since $g$ is locally Lipschitz continuous, so is $h$. Moreover, $[h \leq 0]=g^{-1}(\Lambda)$ where $[h \leq 0]:=\left\{u \in \mathbb{R}^{n}: h(u) \leq 0\right\}$. Theorem 2.1 [8] reveals that $M$ is calm at $(0, \bar{z})$, provided that (11) holds. The proposition is proved.

Remark 5.1 Clearly, if the matrix $B$ is surjective, then it suffices to consider $\Lambda=$ gph $F$ and $g(z)=\left(C z, B^{-1}(\bar{p}-f(z))\right), z \in \mathbb{R}^{n}$. Further, Theorem 2.1 in [8] also 
gives an upper estimate of the corresponding calmness modulus. Namely, if there is $\gamma>0$ such that $\|\xi\| \geq \gamma$ for each $\xi \in \partial_{>} h(\bar{z})$, then the set-valued mapping $M$ from the proof of the above proposition is calm at $(0, \bar{z})$ with modulus not exceeding $1 / \gamma$. Denote by $\varphi$ the mapping defined in (12). A closer look at the proof of Lemma 1 in [7] reveals that $S$ is calm at $(\bar{p}, \bar{z})$ with modulus not exceeding $K / \gamma$, where $K>0$ is the Lipschitz constant of $\varphi$ at $(\bar{p}, \bar{z})$. To be more precise, there is $\varepsilon>0$ such that

$$
\left\|\varphi\left(p^{\prime}, z\right)-\varphi\left(p^{\prime \prime}, z\right)\right\| \leq K\left\|p^{\prime}-p^{\prime \prime}\right\| \quad \text { whenever } p^{\prime}, p^{\prime \prime} \in \mathbb{B}(\bar{p}, \varepsilon), z \in \mathbb{B}(\bar{z}, \varepsilon) .
$$

Remark 5.2 Similar steps, as in the proof of the above theorem, show that the condition $0 \notin \partial h(\bar{z})$ ensures the (stronger) Aubin/Lipschitz-like property of $S$ at the reference point (as observed by one of the referees). However, we prefer to use the well-known Mordukhovich criterion.

The function $h$ is a composite function, so one can try to use some chain rule. For example, since $g(\bar{z}) \in \Lambda$, one may use Proposition 6.4 in [9] (with the closed set $C:=\Lambda$, with the Lipschitz continuous non-negative function $\vartheta:=d(\cdot, \Lambda)$, and the continuously differentiable mapping $F:=g$ ) to get

$$
\partial_{>} h(\bar{z}) \subset \nabla g(\bar{z})^{T} \partial_{>} d(\cdot, \Lambda)(g(\bar{z})) .
$$

However, in our setting this does not seem to be the right way to proceed, since the resulting set can be too large.

Example 5.1 Let $m=n=1, B=C=1$. Suppose that $f: \mathbb{R} \rightarrow \mathbb{R}$ and $F: \mathbb{R} \rightrightarrows \mathbb{R}$ are defined as follows:

$$
f(z):=z, z \in \mathbb{R}, \quad F(z):= \begin{cases}-1-z, & z<0, \\ {[-1,1],} & z=0, \\ 1-z, & z>0 .\end{cases}
$$

Let $\bar{z}:=0$ and $\bar{p}:=-1$. It is easy to verify that both (8) and (10) are violated. So $S$ has neither Aubin nor the isolated calmness property at the reference point. Note that

$$
h(z)=d((z,-1-z,-1-z), \operatorname{gph} F \times \mathbb{R})=d((z,-1-z), \operatorname{gph} F), \quad z \in \mathbb{R} .
$$

Let us compute $\partial_{>} h(0)$. Recall that this set contains those $\xi \in \mathbb{R}$ such that there are sequences $\left(z^{k}\right)_{k \in \mathbb{N}}$ and $\left(\xi^{k}\right)_{k \in \mathbb{N}}$ converging to 0 and $\xi$, respectively, with

$$
h\left(z^{k}\right) \downarrow h(0)=0 \quad \text { as } k \rightarrow \infty \quad \text { and } \quad \xi^{k} \in \partial_{F} h\left(z^{k}\right) \quad \text { for each } k \in \mathbb{N} .
$$

If $z<0$, then the point $(z,-1-z)^{T} \in \operatorname{gph} F$, thus $h(z)=0$, hence we have to consider $\left(z^{k}\right)_{k \in \mathbb{N}}$ in ]0, $\infty$ [ only. Moreover, there is $\delta>0$ such that, for each $\left.z \in\right] 0, \delta[$, the point $(z,-1-z)$ has the unique nearest point $(0,-1)$ in gph $F$. Therefore

$$
\left.h(z)=\sqrt{z^{2}+(-1-z+1)^{2}}=\sqrt{2} z, \quad z \in\right] 0, \delta[.
$$

Hence, $\partial_{F} h(z)=\{\sqrt{2}\}$ for such a point $z$. Let $\left(z^{k}\right)_{k \in \mathbb{N}}$ be any sequence converging to 0 such that $h\left(z^{k}\right) \downarrow 0$ as $k \rightarrow \infty$. We may assume without any loss of generality that 
$\left.z^{k} \in\right] 0, \delta\left[\right.$ for each $k \in \mathbb{N}$. Thus $\partial_{F} h\left(z^{k}\right)=\{\sqrt{2}\}$, hence $\partial_{>} h(0)=\{\sqrt{2}\}$. Therefore $S$ is calm at the reference point with modulus not exceeding $1 / \sqrt{2}$.

To show the failure when one uses the chain rule, let $g: \mathbb{R} \rightarrow \mathbb{R}^{2}$ be for each $z \in \mathbb{R}$ defined by $g(z):=(z,-1-z)^{T}$. Then $\nabla g(0)=(1,-1)^{T}$. As $\bar{y}:=g(0)=(0,-1) \in$ gph $F$, we arrive at the condition

$$
0 \notin\left\{\alpha-\beta:(\alpha, \beta)^{T} \in \partial_{>} d(\cdot, \operatorname{gph} F)(\bar{y})\right\} .
$$

Let $z<0$ be arbitrary. Then the point $(z,-1+z)$ has the unique nearest point $(0,-1)$ in $\operatorname{gph} F$. Hence (3) implies that $(-1,-1)^{T}=(z, z)^{T} /(|z| \sqrt{2})$ is in $\partial_{F} d(\cdot, \operatorname{gph} F)(z,-1+z)$. For each $k \in \mathbb{N}$, put $y^{k}:=(-1 / k,-1-1 / k)$ and $\xi^{k}:=$ $(-1,-1)^{T}$. So we have $y^{k} \rightarrow \bar{y}$ and $d\left(y^{k}, \operatorname{gph} F\right) \downarrow d(\bar{y}, \operatorname{gph} F)=0$ as $k \rightarrow \infty$ with $\xi^{k} \in \partial_{F} d(\cdot, \operatorname{gph} F)\left(y^{k}\right)$ for each $k \in \mathbb{N}$. Thus $\xi:=(-1,-1)^{T}$ is in $\partial_{>} d(\cdot, \operatorname{gph} F)(\bar{y})$. So the above sufficient condition for calmness is not satisfied.

Let $\bar{z}<0$. Then $\bar{p}=-1$ is the only point of $\Phi(\bar{z})$. Again, for each $z \in \mathbb{R}$ we have $h(z)=d((z,-1-z)$, gph $F)$. Then there is $\delta>0$ such that $(z,-1-z)$ is in $\operatorname{gph} F$ whenever $z \in] \bar{z}-\delta, \bar{z}+\delta[$. Thus $h(z)=0$ for such a point $z$. This means that $\partial_{>} h(\bar{z})=\emptyset$ because no sequence $\left(z^{k}\right)_{k \in \mathbb{N}}$ converging to $\bar{z}$ satisfies $h\left(z^{k}\right)>0$ for each $k \in \mathbb{N}$. Consequently, $S$ is calm at the reference point. Note that any such a point $\bar{z}$ is an interior point of $S(-1)$.

\section{Application to Electrical Circuits}

A nonlinear term $f$ in (1) allows often to simplify the set-valued mapping $F$ by splitting it into a single-valued part and (possibly simpler) multivalued one. Then the first mentioned part can be added to $f$. We illustrate this in the following example.

Example 6.1 Consider the circuit in Fig. 1 involving a load resistance $R>0$, a source $E>0$, an input-signal source $u$ and corresponding instantaneous current $i$. Suppose
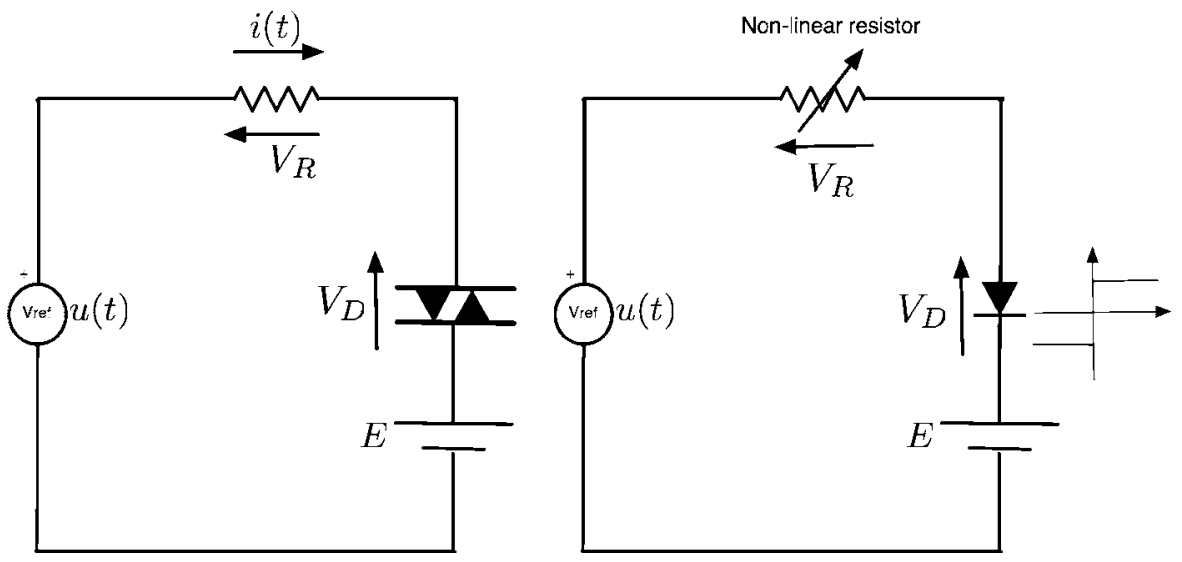

Fig. 1 Two equivalent circuits of Example 6.1 
Fig. 2 I-V characteristic $\Psi$ of a DIAC
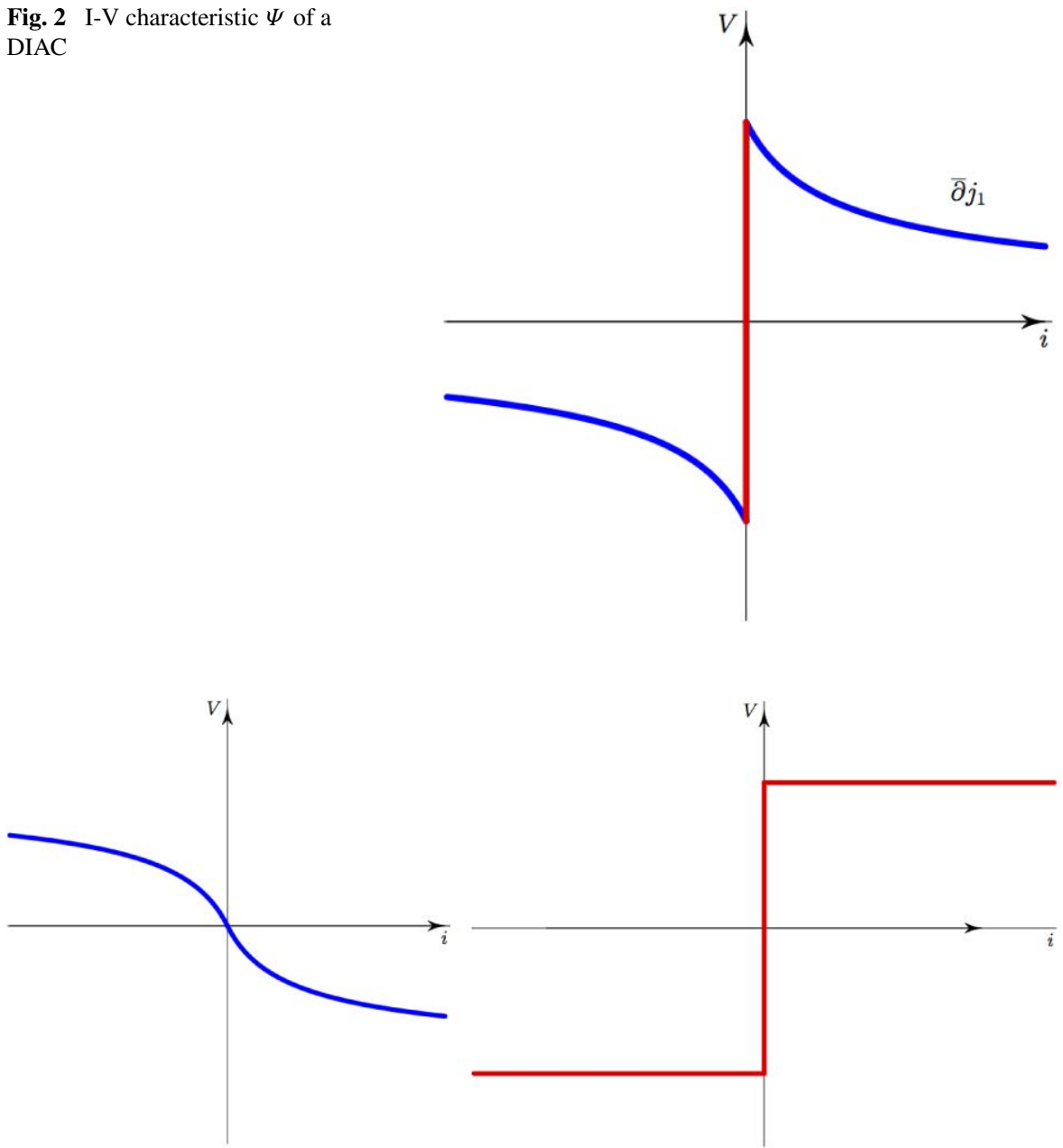

Fig. 3 The mappings $\psi$ and $F$

that the voltage-current characteristic of the DIAC is given by a set-valued mapping $\Psi: \mathbb{R} \rightrightarrows \mathbb{R}$. Assume that $\Psi(0):=[-1,1]$, that $\Psi$ is single-valued and continuously differentiable on $\mathbb{R} \backslash\{0\}$, that its graph is symmetric with respect to the origin, that $-1<\Psi(z)<-1-z$ whenever $z \in]-\infty, 0[$, and finally that $\Psi(0-)=-1$ and $\Psi^{\prime}(0-)=-a$ for some $a>0$ (see Fig. 2). Kirchhoff's voltage law reveals that

$$
u-E \in \underbrace{R i}_{V_{R}}+\underbrace{\Psi(i)}_{V_{D}} .
$$

Assume that $R=1$. Putting $F=\partial|\cdot|$, one infers that there is a continuously differentiable odd function $\psi: \mathbb{R} \rightarrow \mathbb{R}$ such that $\Psi=\psi+F$. Moreover, $\psi(0)=0$, $\psi^{\prime}(0)=-a$, and $0<\psi(z)<-z$ whenever $z<0$ (see Fig. 3). Putting $z:=i$ and 

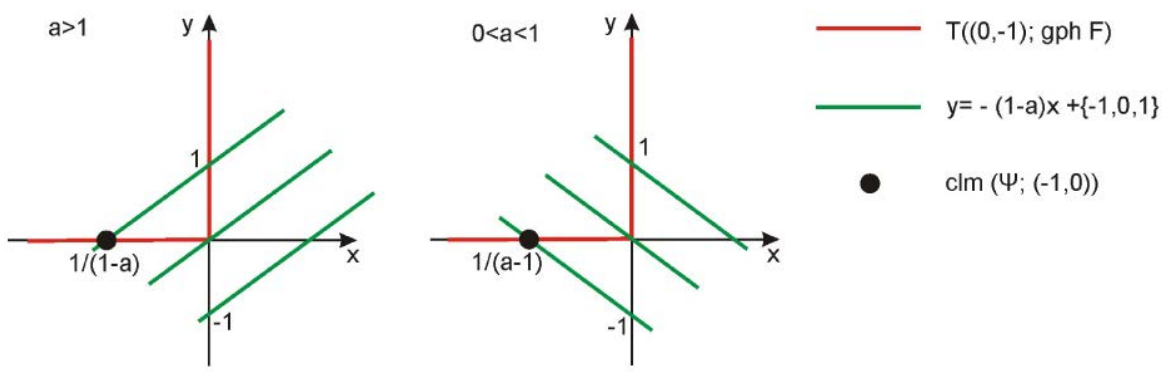

Fig. 4 Calmness modulus from Example 6.1 (Color figure online)

$p:=u-E$ one arrives at (1) with $m=n=1, B=C=1$, and $f$ defined for each $z \in \mathbb{R}$ by $f(z):=z+\psi(z)$.

Let $(\bar{z}, \bar{p})=(0,-1) \in \operatorname{gph} \Phi$. In [1, Example 5.1] it is proved that $S$ has Aubin/Lipschitz-like property (isolated calmness property) at $(\bar{p}, \bar{z})$ if and only if $0<a<1(a \neq 1)$. There it is also shown that $\operatorname{lip}(S ;(\bar{p}, \bar{z}))=1 /(1-a)$. First, to illustrate our approach, let us use Corollary 4.1 to check isolated calmness and Theorem 4.1 to compute the calmness modulus (which was not done in [1]).

Note that $f^{\prime}(\bar{z})=1-a$ and $\bar{v}=-1$. A simple calculation yields that

$$
T((0,-1) ; \operatorname{gph} F)=\mathbb{R}_{+}\left(\begin{array}{l}
0 \\
1
\end{array}\right) \cup \mathbb{R}_{+}\left(\begin{array}{c}
-1 \\
0
\end{array}\right) .
$$

Thus, the necessary and sufficient condition (10) for the isolated calmness property of $S$ at $(\bar{p}, \bar{z})$ with $b \in \mathbb{R}$ is

$$
(b,-(1-a) b)^{T} \in T((0,-1) ; \operatorname{gph} F) \quad \Longrightarrow \quad b=0 .
$$

In $x y$-plane (see Fig. 4), this means that the intersection of the above tangent cone with the graph of the function $y=-(1-a) x, x \in \mathbb{R}$, is a singleton containing the origin only. Clearly, this is the case if and only if $a \neq 1$. To compute $\operatorname{clm}(S ;(\bar{p}, \bar{z}))$ one has to find a maximum of $|x|$ subject to the constraint $-1 \leq(1-a) x+y \leq 1$ with $(x, y) \in T((0,-1)$; gph $F)$. Which immediately gives (see Fig. 4) that

$$
\operatorname{clm}(S ;(\bar{p}, \bar{z}))= \begin{cases}\frac{1}{|a-1|}, & \text { if } a \neq 1, \\ \infty, & \text { otherwise }\end{cases}
$$

So, if $a=1$, then $S$ has neither Aubin nor isolated calmness property at $(\bar{p}, \bar{z})$. Note that the sufficient condition (11) for calmness property is not satisfied in this case. Indeed, $(\bar{z}, \bar{p}) \in \operatorname{gph} F \cap \operatorname{gph} \Phi$ and $h: \mathbb{R} \rightarrow \mathbb{R}_{+}$is for each $z \in \mathbb{R}$ defined by

$$
h(z):=d((z,-1-z-\psi(z)), \operatorname{gph} F) .
$$

Note that $-1-z-\psi(z)<-1-z$ and $\psi(z)<-z$ whenever $z<0$. Given $z<0$, the point $(z,-1-z-\psi(z))^{T}$ has the unique nearest point $(z,-1)^{T}$ in gph $F$. Therefore $h(z)=|z+\psi(z)|=-z-\psi(z)$. 
Fig. 5 Circuit of Example 6.2

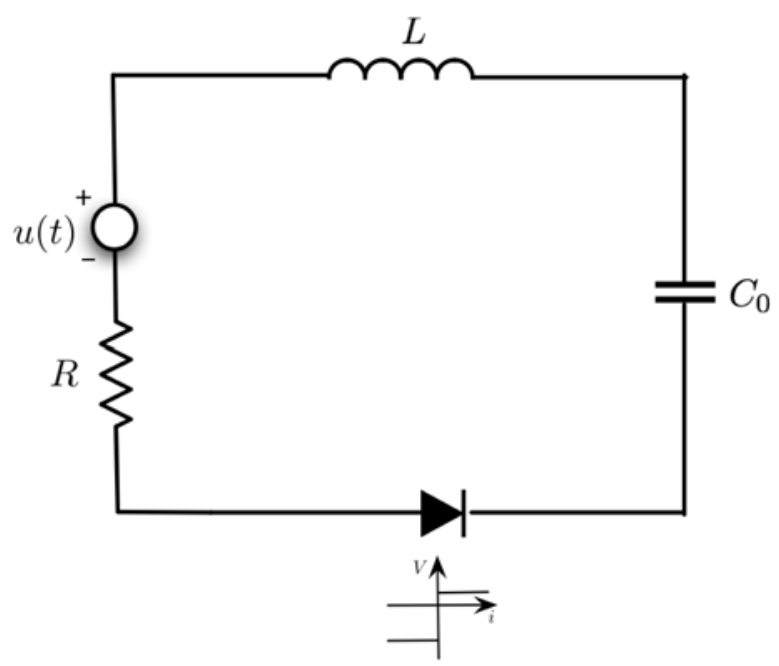

Hence, $\partial_{F} h(z)=\left\{-1-\psi^{\prime}(z)\right\}$ whenever $z<0$. For each $k \in \mathbb{N}$, put $z^{k}:=-\frac{1}{k}$ and $\xi^{k}:=-1-\psi^{\prime}\left(z^{k}\right)$. Then both $\left(z^{k}\right)_{k \in \mathbb{N}}$ and $\left(\xi^{k}\right)_{k \in \mathbb{N}}$ converge to 0 . For any $k \in \mathbb{N}$, we have $\xi^{k} \in \partial_{F} h\left(z^{k}\right)$, and $\psi\left(z^{k}\right)<-z^{k}$ implies that $-1-z^{k}-\psi\left(z^{k}\right)+1$ is positive, so $h\left(z^{k}\right)>0$. Thus $0 \in \partial_{>} h(0)$.

However, $S$ is not calm at $(-1,0)$. Indeed, suppose on the contrary that it is. As $S(-1)=\left\{z \in \mathbb{R}:(z,-1-z-\psi(z))^{T} \in \operatorname{gph} F\right\}=\{0\}$, the point 0 is an isolated point of $S(-1)$, thus $S$ would have the isolated calmness property at $(-1,0)$, which is not the case as seen before.

Example 6.2 Let us consider the circuit depicted in Fig. 5 involving a load resistance $R>0$, an input-signal source $u$ and corresponding instantaneous current $i$, an inductor with inductance $L>0$, a capacitor with capacitance $C_{0}>0$, and a diode with the voltage-current characteristics given for some $\alpha_{1}<0<\alpha_{2}$ by

$$
F(x):= \begin{cases}\alpha_{1}, & x<0, \\ {\left[\alpha_{1}, \alpha_{2}\right],} & x=0, \\ \alpha_{2}, & x>0 .\end{cases}
$$

Using the Kirchhoff voltage law, we have

$$
u-U_{R}-U_{L}-U_{C_{0}}=U_{D},
$$

where $U_{R}=R i, U_{L}=L \frac{\mathrm{d} i}{\mathrm{~d} t}, U_{C_{0}}=\frac{1}{C_{0}} \int i(t) \mathrm{d} t$ and $U_{D} \in-F(-i)$ denote the difference of potential across the resistor, the inductor, the capacitor, and the diode, respectively. Setting

$$
z_{1}:=\int i \mathrm{~d} t \quad \text { and } \quad z_{2}:=\dot{z}_{1} \quad(=i)
$$


we have

$$
L \dot{z}_{2}=-\frac{1}{C_{0}} z_{1}-R z_{2}+u-U_{D}
$$

Hence, dividing by $L$, we arrive at the dynamic system

$$
\left(\begin{array}{l}
\dot{z}_{1} \\
\dot{z}_{2}
\end{array}\right)=\left(\begin{array}{cc}
0 & 1 \\
-\frac{1}{L C_{0}} & -\frac{R}{L}
\end{array}\right)\left(\begin{array}{l}
z_{1} \\
z_{2}
\end{array}\right)+\left(\begin{array}{c}
0 \\
\frac{1}{L}
\end{array}\right) u+\left(\begin{array}{c}
0 \\
-\frac{1}{L}
\end{array}\right) U_{D}
$$

with

$$
U_{D} \in-F\left((0-1)\left(\begin{array}{l}
z_{1} \\
z_{2}
\end{array}\right)\right) .
$$

From now on, assume that $R=L=C_{0}=1$, that $\alpha_{1}=-100$, and that $\alpha_{2}=1$. The steady states of the above dynamic system may be viewed as particular solutions to (1) with $n=2, m=1$,

$$
A:=\left(\begin{array}{cc}
0 & 1 \\
-1 & -1
\end{array}\right), \quad B:=\left(\begin{array}{l}
0 \\
1
\end{array}\right), \quad C:=\left(\begin{array}{ll}
0 & -1
\end{array}\right),
$$

and

$$
f(z):=A z, z \in \mathbb{R}^{2} \quad \text { and } \quad F(x):= \begin{cases}-100, & x<0, \\ {[-100,1],} & x=0, \\ 1, & x>0 .\end{cases}
$$

So, for $z=\left(z_{1}, z_{2}\right)^{T} \in \mathbb{R}^{2}$ and $p=\left(p_{1}, p_{2}\right)^{T} \in \mathbb{R}^{2}$, (1) reads as

$$
p_{1}=z_{2}, \quad p_{2} \in-z_{1}-z_{2}+F\left(-z_{2}\right) \text {. }
$$

Moreover, $C C^{T}=B^{T} B=1$ and $\operatorname{rge} B=\operatorname{rge} C^{T}=\{0\} \times \mathbb{R}$. Let $(\bar{z}, \bar{p}) \in \operatorname{gph} \Phi$ be arbitrary. The necessary and sufficient condition (8) for Aubin/Lipschitz-like property of $S$ at $(\bar{p}, \bar{z})$ with $\xi=\left(\xi_{1}, \xi_{2}\right)^{T} \in \mathbb{R}^{2}$ is

$$
\left.\begin{array}{l}
\left(\xi_{2}-\xi_{1}, \xi_{2}\right)^{T} \in-N((C \bar{z}, \bar{v}) ; \operatorname{gph} F) \\
\left(-\xi_{2}, \xi_{1}-\xi_{2}\right)^{T} \in\{0\} \times \mathbb{R} .
\end{array}\right\} \quad \Longrightarrow \quad \xi_{1}=0 \quad \text { and } \quad \xi_{2}=0
$$

The necessary and sufficient condition (10) for the isolated calmness property of $S$ at $(\bar{p}, \bar{z})$ with $b=\left(b_{1}, b_{2}\right)^{T} \in \mathbb{R}^{2}$ is

$$
\left.\begin{array}{l}
\left(-b_{2}, b_{1}+b_{2}\right)^{T} \in T((C \bar{z}, \bar{v}) ; \operatorname{gph} F) \\
\left(b_{2},-b_{1}-b_{2}\right)^{T} \in\{0\} \times \mathbb{R}
\end{array}\right\} \Rightarrow b_{1}=0 \quad \text { and } \quad b_{2}=0
$$

Note that the latter inclusion in (13) and (14) immediately implies that $\xi_{2}=0$ and $b_{2}=0$, respectively. First, consider five particular cases (see Fig. 6). 


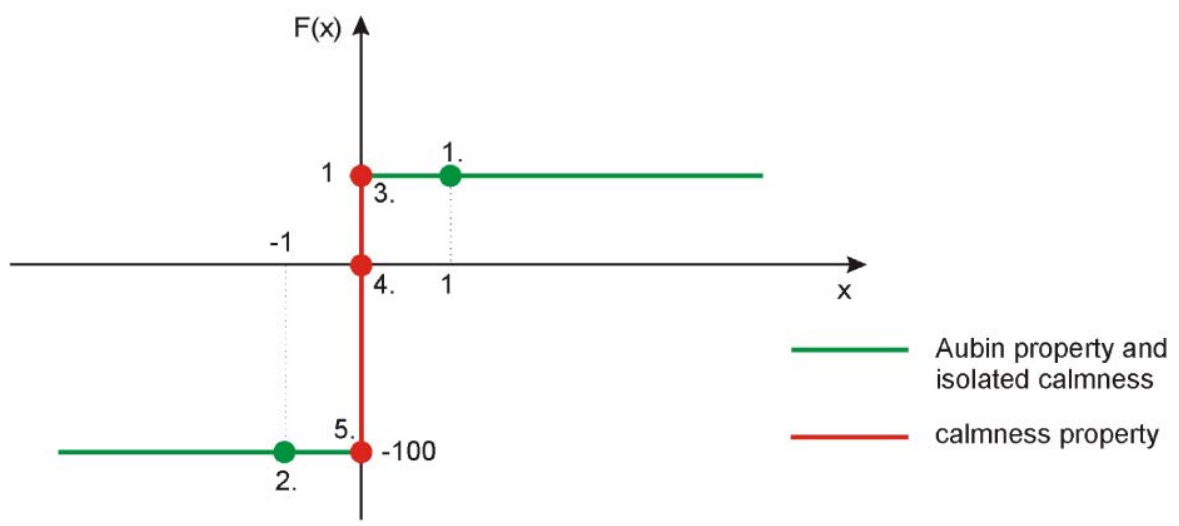

Fig. $6 F$ from Example 6.2 (Color figure online)

1. Let $\bar{z}:=(0,-1)^{T}$ and $\bar{p}:=(-1,2)^{T}$.

Hence $C \bar{z}=1, \bar{v}=1$, and

$$
T((1,1) ; \operatorname{gph} F)=\mathbb{R}\left(\begin{array}{l}
1 \\
0
\end{array}\right) \quad \text { and } \quad N((1,1) ; \operatorname{gph} F)=\mathbb{R}\left(\begin{array}{l}
0 \\
1
\end{array}\right) .
$$

Clearly, (13) is satisfied. So $S$ has the Aubin/Lipschitz-like property (hence it has the calmness property) at $(\bar{p}, \bar{z})$. Moreover, (14) holds as well. Thus $S$ has the isolated calmness property at $(\bar{p}, \bar{z})$.

2. Let $\bar{z}:=(0,1)^{T}$ and $\bar{p}:=(1,-101)^{T}$.

Hence $C \bar{z}=-1$ and $\bar{v}=100$. Moreover, $T((-1,100)$; gph $F)=T((1,1)$; gph $F)$ and $N((-1,100) ; \operatorname{gph} F)=N((1,1) ; \operatorname{gph} F)$. In view of the previous case, we may conclude that $S$ has the Aubin/Lipschitz-like property and the isolated calmness property at $(\bar{p}, \bar{z})$.

3. Let $\bar{z}:=(0,0)^{T}$ and $\bar{p}:=(0,1)^{T}$.

Then $C \bar{z}=0$ and $\bar{v}=1$. A simple calculation reveals that the tangent cone $T((0,1) ; \operatorname{gph} F)$ and the normal cone $N((0,1) ; \operatorname{gph} F)$ are equal to

$$
\mathbb{R}_{+}\left(\begin{array}{c}
0 \\
-1
\end{array}\right) \cup \mathbb{R}_{+}\left(\begin{array}{l}
1 \\
0
\end{array}\right) \text { and } \mathbb{R}\left(\begin{array}{c}
-1 \\
0
\end{array}\right) \cup \mathbb{R}\left(\begin{array}{l}
0 \\
1
\end{array}\right) \cup \text { cone }\left\{\left(\begin{array}{c}
-1 \\
0
\end{array}\right),\left(\begin{array}{l}
0 \\
1
\end{array}\right)\right\},
$$

respectively. Clearly, (13) is violated (take $\xi_{1}:=1$ and $\xi_{2}:=0$ ). So $S$ does not have the Aubin/Lipschitz-like property. Moreover, (14) is violated too (take $b_{1}:=-1$ and $\left.b_{2}:=0\right)$. Thus $S$ does not have the isolated calmness property at $(\bar{p}, \bar{z})$.

To check the calmness property, we are going to apply Proposition 5.1. Note that $\Lambda=\operatorname{gph} F \times\{0\} \times \mathbb{R}$. The functions $g: \mathbb{R}^{2} \rightarrow \mathbb{R}^{2} \times \mathbb{R}^{2}$ and $h: \mathbb{R}^{2} \rightarrow \mathbb{R}_{+}$are for each $z=\left(z_{1}, z_{2}\right)^{T} \in \mathbb{R}^{2}$ defined by $g(z)=\left(-z_{2}, 1+z_{1}+z_{2},-z_{2}, 1+z_{1}+z_{2}\right)^{T}$ and

$$
h(z)=d(g(z), \Lambda)=d\left(\left(-z_{2}, 1+z_{1}+z_{2},-z_{2}, 1+z_{1}+z_{2}\right)^{T}, \operatorname{gph} F \times\{0\} \times \mathbb{R}\right),
$$


respectively. Hence, we need to prove that $(0,0)^{T} \notin \partial_{>} h(\bar{z})$. Without any loss of generality, assume that the norm on $\mathbb{R}^{2} \times \mathbb{R}^{2}$ is for each its point $(u, v)$ given by $\|(u, v)\|=\|u\|_{2}+\|v\|_{2}$, where $\|\cdot\|_{2}$ is Euclidean norm on $\mathbb{R}^{2}$.

First, we compute $\partial d(\cdot, \operatorname{gph} F)(u)$ for each $u=(x, y)^{T} \in \mathbb{R}^{2} \backslash \operatorname{gph} F$ sufficiently close to $\bar{u}:=(0,1)^{T}$. In view of (2), it suffices to find nearest points to $u$ in gph $F$. This yields $r \in] 0,1$ [ such that for each $u=(x, y)^{T} \in \mathbb{B}_{2}(\bar{u}, r)$ one has

$$
\partial d(\cdot, \operatorname{gph} F)(u)= \begin{cases}\left\{(0,1)^{T}\right\}, & \text { if } x \geq 0, y>1, \\ \left\{(-1,0)^{T}\right\}, & \text { if } x<0, y \leq 1, \\ \left\{\frac{1}{\sqrt{x^{2}+(y-1)^{2}}}(x, y-1)^{T}\right\}, & \text { if } x<0, y>1, \\ \left\{(0,-1)^{T}\right\}, & \text { if } x>0,1>y>1-x, \\ \left\{(1,0)^{T}\right\}, & \text { if } x>0, y<1-x, \text { and } \\ \left\{(1,0)^{T},(0,-1)^{T}\right\}, & \text { if } x>0, y=1-x .\end{cases}
$$

Second, we claim that given $z=\left(z_{1}, z_{2}\right)^{T} \in \mathbb{R}^{2}$ and $\eta \in \mathbb{R}^{2}$ such that $h(z)>0$, that $\bar{p}-A z \in \mathbb{B}_{2}(\bar{u}, r)$, and that $\eta \in \partial h(z)$ one has $\|\eta\| \geq 1$. Indeed,

$$
\begin{aligned}
h(z) & =d\left(\left(-z_{2}, 1+z_{1}+z_{2}\right)^{T}, \operatorname{gph} F\right)+d\left(\left(-z_{2}, 1+z_{1}+z_{2}\right)^{T},\{0\} \times \mathbb{R}\right) \\
& =d\left(\left(-z_{2}, 1+z_{1}+z_{2}\right)^{T}, \operatorname{gph} F\right)+\left|z_{2}\right|=d(\bar{p}-A z, \operatorname{gph} F)+\left|z_{2}\right| .
\end{aligned}
$$

Since, $h$ is the sum of two Lipschitz functions, Theorem 3.36 in [2] implies that

$$
\partial h(z) \subset \partial d(\bar{p}-A(\cdot), \operatorname{gph} F)(z)+\{0\} \times \partial|\cdot|\left(z_{2}\right) .
$$

As $A$ is surjective, Proposition 1.112(i) in [2] reveals that

$$
\partial d(\bar{p}-A(\cdot), \operatorname{gph} F)(z)=-A^{T} \partial d(\cdot, \operatorname{gph} F)\left(-z_{2}, 1+z_{1}+z_{2}\right) .
$$

Collecting the above partial results we are in position to compute an upper estimate of $\partial h(z)$. Recall that $h(z)>0$, thus it suffices to consider the following cases.

(i) If $z_{2}=0$, then $z_{1}>0$ because $1+z_{1} \notin F(0)$ (the case $z_{1}<-101$ is impossible as $\left.\bar{p}-A z=\left(0,1+z_{1}\right)^{T} \in \mathbb{B}_{2}(\bar{u}, r)\right)$. Thus

$$
\partial h(z) \subset\left(\begin{array}{cc}
0 & 1 \\
-1 & 1
\end{array}\right)\left(\begin{array}{l}
0 \\
1
\end{array}\right)+\left(\begin{array}{c}
0 \\
{[-1,1]}
\end{array}\right)=\left(\begin{array}{c}
1 \\
{[0,2]}
\end{array}\right) .
$$

(ii) If $z_{2}<0$ and $1+z_{1}+z_{2}>1$, then

$$
\partial h(z) \subset\left(\begin{array}{cc}
0 & 1 \\
-1 & 1
\end{array}\right)\left(\begin{array}{l}
0 \\
1
\end{array}\right)+\left(\begin{array}{c}
0 \\
-1
\end{array}\right)=\left(\begin{array}{l}
1 \\
0
\end{array}\right) .
$$

(iii) If $z_{2}<0$ and $1+z_{1}+z_{2}<1$, then

$$
\partial h(z) \subset\left(\begin{array}{cc}
0 & 1 \\
-1 & 1
\end{array}\right)\left\{\left(\begin{array}{c}
0 \\
-1
\end{array}\right),\left(\begin{array}{l}
1 \\
0
\end{array}\right)\right\}+\left(\begin{array}{c}
0 \\
-1
\end{array}\right)=\left\{\left(\begin{array}{c}
-1 \\
-2
\end{array}\right),\left(\begin{array}{c}
0 \\
-2
\end{array}\right)\right\} .
$$


(iv) If $z_{2}>0$ and $1+z_{1}+z_{2} \leq 1$, then

$$
\partial h(z) \subset\left(\begin{array}{cc}
0 & 1 \\
-1 & 1
\end{array}\right)\left(\begin{array}{c}
-1 \\
0
\end{array}\right)+\left(\begin{array}{l}
0 \\
1
\end{array}\right)=\left(\begin{array}{l}
0 \\
2
\end{array}\right) .
$$

(v) If $z_{2}>0$ and $1+z_{1}+z_{2}>1$, then

$$
\begin{aligned}
\partial h(z) & \subset \frac{1}{\sqrt{z_{2}^{2}+\left(z_{1}+z_{2}\right)^{2}}}\left(\begin{array}{cc}
0 & 1 \\
-1 & 1
\end{array}\right)\left(\begin{array}{c}
-z_{2} \\
z_{1}+z_{2}
\end{array}\right)+\left(\begin{array}{l}
0 \\
1
\end{array}\right) \\
& =\left(\begin{array}{c}
\frac{z_{1}+z_{2}}{\sqrt{z_{2}^{2}+\left(z_{1}+z_{2}\right)^{2}}} \\
\frac{z_{1}+2 z_{2}}{\sqrt{z_{2}^{2}+\left(z_{1}+z_{2}\right)^{2}}}+1
\end{array}\right) .
\end{aligned}
$$

In any case, $\|\eta\| \geq 1$ for each $\eta \in \partial h(z)$, the claim is proved.

To conclude, pick an arbitrary $\xi \in \partial_{>} h(\bar{z})$ if there is any. Find a sequence $z^{k}=$ $\left(z_{1}^{k}, z_{2}^{k}\right)^{T}$ in $\mathbb{R}^{2}$ converging to $\bar{z}=(0,0)^{T}$ along with a sequence $\left(\xi^{k}\right)_{k \in \mathbb{N}}$ converging to $\xi$ such that

$$
h\left(z^{k}\right) \downarrow h(\bar{z})=0 \quad \text { as } k \rightarrow \infty \quad \text { and } \quad \xi^{k} \in \partial h\left(z^{k}\right) \quad \text { for each } k \in \mathbb{N} .
$$

As $\bar{p}=\bar{u}$, there is $k_{0} \in \mathbb{N}$ such that $\bar{p}-A z^{k} \in \mathbb{B}_{2}(\bar{u}, r)$ whenever $k>k_{0}$. So, the previous claim implies that $\|\xi\| \geq 1$. In particular, $(0,0)^{T} \notin \partial_{>} h(\bar{z})$. Thus $S$ is calm at $(\bar{p}, \bar{z})$ with the calmness modulus not exceeding 2 .

4. Let $\bar{z}:=(0,0)^{T}$ and $\bar{p}:=(0,0)^{T}$.

Therefore $C \bar{z}=0$ and $\bar{v}=0$. Moreover,

$$
T((0,0) ; \operatorname{gph} F)=\mathbb{R}\left(\begin{array}{l}
0 \\
1
\end{array}\right) \quad \text { and } \quad N((0,0) ; \operatorname{gph} F)=\mathbb{R}\left(\begin{array}{l}
1 \\
0
\end{array}\right) .
$$

Similarly to the previous case, one infers that $S$ has neither the Aubin/Lipschitz-like nor the isolated calmness property at $(\bar{p}, \bar{z})$.

To check the calmness, note that for each $z=\left(z_{1}, z_{2}\right)^{T} \in \mathbb{R}^{2}$ we have $g(z)=$ $\left(-z_{2}, z_{1}+z_{2},-z_{2}, z_{1}+z_{2}\right)^{T}$ and

$$
h(z)=d\left(\left(-z_{2}, z_{1}+z_{2},-z_{2}, z_{1}+z_{2}\right)^{T}, \operatorname{gph} F \times\{0\} \times \mathbb{R}\right) .
$$

First, find again $r \in] 0,1\left[\operatorname{such}\right.$ that $\partial d(\cdot, \operatorname{gph} F)(u)=\left\{\left(\frac{x}{|x|}, 0\right)^{T}\right\}$ whenever $u=$ $(x, y)^{T} \in \mathbb{B}_{2}(\bar{u}, r)$ with $\bar{u}:=(0,0)^{T}$.

Second, we claim that given $z=\left(z_{1}, z_{2}\right)^{T} \in \mathbb{R}^{2}$ and $\eta \in \mathbb{R}^{2}$ such that $h(z)>0$, that $-A z \in \mathbb{B}_{2}(\bar{u}, r)$, and that $\eta \in \partial h(z)$ one has $\|\eta\| \geq 2$. Indeed,

$$
h(z)=d\left(\left(-z_{2}, z_{1}+z_{2}\right)^{T}, \operatorname{gph} F\right)+\left|z_{2}\right|=d(-A z, \operatorname{gph} F)+\left|z_{2}\right| .
$$

and $\partial h(z) \subset-A^{T} \partial d(\cdot, \operatorname{gph} F)(-A z)+\{0\} \times \partial|\cdot|\left(z_{2}\right)$. Suppose that $z_{2}=0$, then the assumption $h(z)>0$ implies that $z_{1} \notin F(0)$, so either $z_{1}>1$ or $z_{1}<-100$. Both the 
cases are impossible because $\left|z_{1}\right| \leq r<1$. Therefore $z_{2} \neq 0$. Moreover, for $u:=-A z$ we get either

$$
\partial h(z) \subset\left(\begin{array}{cc}
0 & 1 \\
-1 & 1
\end{array}\right)\left(\begin{array}{c}
-1 \\
0
\end{array}\right)+\left(\begin{array}{l}
0 \\
1
\end{array}\right)=\left(\begin{array}{l}
0 \\
2
\end{array}\right) \quad \text { when } z_{2}>0,
$$

or

$$
\partial h(z) \subset\left(\begin{array}{cc}
0 & 1 \\
-1 & 1
\end{array}\right)\left(\begin{array}{l}
1 \\
0
\end{array}\right)+\left(\begin{array}{c}
0 \\
-1
\end{array}\right)=\left(\begin{array}{c}
0 \\
-2
\end{array}\right) \quad \text { when } z_{2}<0 .
$$

In any case, $\|\eta\| \geq 2$ for each $\eta \in \partial h(z)$ which establishes the claim.

As in the previous case, the claim yields that $\|\xi\| \geq 2$ whenever $\xi \in \partial_{>} h(\bar{z})$. Thus $S$ is calm at $(\bar{p}, \bar{z})$ with the calmness modulus not exceeding 1 .

5. Let $\bar{z}=(0,0)^{T}$ and $\bar{p}=(0,-100)^{T}$.

Then $C \bar{z}=0$ and $\bar{v}=-100$. A simple calculation reveals that the tangent cone $T((0,-100)$; gph $F)$ and the normal cone $N((0,-100)$; gph $F)$ are equal to

$$
\mathbb{R}_{+}\left(\begin{array}{c}
-1 \\
0
\end{array}\right) \cup \mathbb{R}_{+}\left(\begin{array}{l}
0 \\
1
\end{array}\right) \quad \text { and } \quad \mathbb{R}\left(\begin{array}{c}
0 \\
-1
\end{array}\right) \cup \mathbb{R}\left(\begin{array}{l}
1 \\
0
\end{array}\right) \cup \operatorname{cone}\left\{\left(\begin{array}{c}
0 \\
-1
\end{array}\right),\left(\begin{array}{l}
1 \\
0
\end{array}\right)\right\}
$$

respectively. Similarly to the previous cases, one infers that $S$ has neither the Aubin/Lipschitz-like nor the isolated calmness property at $(\bar{p}, \bar{z})$.

To check the calmness, note that for each $z=\left(z_{1}, z_{2}\right)^{T} \in \mathbb{R}^{2}$ we have $g(z)=$ $\left(-z_{2},-100+z_{1}+z_{2},-z_{2},-100+z_{1}+z_{2}\right)^{T}$ and

$$
h(z)=d\left(\left(-z_{2},-100+z_{1}+z_{2},-z_{2},-100+z_{1}+z_{2}\right)^{T}, \operatorname{gph} F \times\{0\} \times \mathbb{R}\right) .
$$

We shall use the same steps as in the case 3. First, find $r \in] 0,1[$ such that for each $u=(x, y)^{T} \in \mathbb{B}_{2}(\bar{u}, r)$ with $\bar{u}:=(0,-100)^{T}$ one has $\partial d(\cdot, \operatorname{gph} F)(u)$ equal to

$$
\begin{cases}\left\{(1,0)^{T}\right\}, & \text { if } x>0, y \geq-100, \\ \left\{(0,-1)^{T}\right\}, & \text { if } x \leq 0, y<-100, \\ \left\{\frac{1}{\sqrt{x^{2}+(y+100)^{2}}}(x, y+100)^{T}\right\}, & \text { if } x>0, y<-100, \\ \left\{(0,1)^{T}\right\}, & \text { if } x<0,-100<y<-100-x, \\ \left\{(-1,0)^{T}\right\}, & \text { if } x<0, y>-100-x, \text { and } \\ \left\{(-1,0)^{T},(0,1)^{T}\right\}, & \text { if } x<0, y=-100-x .\end{cases}
$$

Second, we claim that given $z=\left(z_{1}, z_{2}\right)^{T} \in \mathbb{R}^{2}$ and $\eta \in \mathbb{R}^{2}$ such that $h(z)>0$, that $\bar{p}-A z \in \mathbb{B}_{2}(\bar{u}, r)$, and that $\eta \in \partial h(z)$ one has $\|\eta\| \geq 1$. Indeed,

$$
h(z)=d\left(\left(-z_{2},-100+z_{1}+z_{2}\right)^{T}, \operatorname{gph} F\right)+\left|z_{2}\right|=d(\bar{p}-A z, \operatorname{gph} F)+\left|z_{2}\right|
$$

and $\partial h(z) \subset-A^{T} \partial d(\cdot, \operatorname{gph} F)\left(-z_{2},-100+z_{1}+z_{2}\right)+\{0\} \times \partial|\cdot|\left(z_{2}\right)$. Consider the following cases: 
(i) If $z_{2}=0$, then $z_{1}<0$ because $-100+z_{1} \notin F(0)$ (the case $z_{1}>101$ is impossible as $\left.\bar{p}-A z=\left(0,-100+z_{1}\right)^{T} \in \mathbb{B}_{2}(\bar{u}, r)\right)$. Thus

$$
\partial h(z) \subset\left(\begin{array}{cc}
0 & 1 \\
-1 & 1
\end{array}\right)\left(\begin{array}{c}
0 \\
-1
\end{array}\right)+\left(\begin{array}{c}
0 \\
{[-1,1]}
\end{array}\right)=\left(\begin{array}{c}
-1 \\
{[-2,0]}
\end{array}\right) .
$$

(ii) If $z_{2}<0$ and $-100+z_{1}+z_{2} \geq-100$, then

$$
\partial h(z) \subset\left(\begin{array}{cc}
0 & 1 \\
-1 & 1
\end{array}\right)\left(\begin{array}{l}
1 \\
0
\end{array}\right)+\left(\begin{array}{c}
0 \\
-1
\end{array}\right)=\left(\begin{array}{c}
0 \\
-2
\end{array}\right) \text {. }
$$

(iii) If $z_{2}<0$ and $-100+z_{1}+z_{2}<-100$, then

$$
\begin{aligned}
\partial h(z) & \subset \frac{1}{\sqrt{z_{2}^{2}+\left(z_{1}+z_{2}\right)^{2}}}\left(\begin{array}{cc}
0 & 1 \\
-1 & 1
\end{array}\right)\left(\begin{array}{c}
-z_{2} \\
z_{1}+z_{2}
\end{array}\right)+\left(\begin{array}{c}
0 \\
-1
\end{array}\right) \\
& =\left(\begin{array}{c}
\frac{z_{1}+z_{2}}{\sqrt{z_{2}^{2}+\left(z_{1}+z_{2}\right)^{2}}} \\
\frac{z_{1}+2 z_{2}}{\sqrt{z_{2}^{2}+\left(z_{1}+z_{2}\right)^{2}}}-1
\end{array}\right) .
\end{aligned}
$$

(iv) If $z_{2}>0$ and $-100+z_{1}+z_{2}<-100$, then

$$
\partial h(z) \subset\left(\begin{array}{cc}
0 & 1 \\
-1 & 1
\end{array}\right)\left(\begin{array}{c}
0 \\
-1
\end{array}\right)+\left(\begin{array}{l}
0 \\
1
\end{array}\right)=\left(\begin{array}{c}
-1 \\
0
\end{array}\right) \text {. }
$$

(v) If $z_{2}>0$ and $-100+z_{1}+z_{2}>-100$, then

$$
\partial h(z) \subset\left(\begin{array}{cc}
0 & 1 \\
-1 & 1
\end{array}\right)\left\{\left(\begin{array}{c}
-1 \\
0
\end{array}\right),\left(\begin{array}{l}
0 \\
1
\end{array}\right)\right\}+\left(\begin{array}{l}
0 \\
1
\end{array}\right)=\left\{\left(\begin{array}{l}
0 \\
2
\end{array}\right),\left(\begin{array}{l}
1 \\
2
\end{array}\right)\right\} .
$$

In any case, $\|\eta\| \geq 1$ for each $\eta \in \partial h(z)$. Indeed, this needs an explanation in case (iii) only. If $z_{2}<0$, then $z_{1}+2 z_{2}<z_{1}+z_{2}<0$. So we may estimate

$$
\frac{\left(z_{1}+z_{2}\right)^{2}}{z_{2}^{2}+\left(z_{1}+z_{2}\right)^{2}}+\frac{\left(z_{1}+2 z_{2}\right)^{2}}{z_{2}^{2}+\left(z_{1}+z_{2}\right)^{2}}-2 \frac{z_{1}+2 z_{2}}{\sqrt{z_{2}^{2}+\left(z_{1}+z_{2}\right)^{2}}}+1 \geq 1 .
$$

The claim is proved.

As in the case 3 , the claim yields that $\|\xi\| \geq 1$ whenever $\xi \in \partial_{>} h(\bar{z})$. Thus $S$ is calm at $(\bar{p}, \bar{z})$ with the calmness modulus not exceeding 2 .

A closer look at the above discussion reveals that any other $(\bar{z}, \bar{p}) \in \operatorname{gph} x \Phi$ fits in one of the above categories and that this depends only on the position of the point $(C \bar{z}, \bar{v})$ in the graph of $F$ (see Fig. 6). This is clear if the point in question belongs to the first two categories. In the remaining ones one has to remember that $C \bar{z}=\bar{z}_{2}=$ $\bar{p}_{1}=0$ and $\left(C z, B^{T}(\bar{p}-A z)\right)^{T}=\bar{p}-A z$ whenever $z=\left(z_{1}, z_{2}\right)^{T} \in \mathbb{R}^{2}$.

Remark 6.1 Suppose that the diode in the above circuit is replaced with a DIAC. The computation in the Example 6.2 shows that one can use the approach from Example 6.1 to establish the calmness property. 


\section{Conclusions}

In this paper, we studied the quantitative stability of a generalized equation involving a general set-valued map. The key point in our analysis is the coderivative criterion for the Aubin/Lipschitz-like property, the graphical derivative for the isolated calmness and the outer subdifferential sufficient condition for the calmness. We applied our theoretical results to the theory of non-regular electrical circuits involving electronic devices like ideal diode, practical diode, and DIAC. Some other nonlinear elements like SCR and transistor could be studied in the same way. In order to apply the calculus rules, the injectivity of the matrix $B$ is assumed. It would be interesting to relax this condition. As observed in Example 6.2, the sufficient condition for the calmness is not easy to check even for one or two dimensional problems. It would be interesting to prove a point-based condition which will be easier to check in practice. Another interesting part would be the simulation of the circuits studied in this paper. This is out of the scope of the current paper and will be the subject of another work.

Acknowledgements The authors would like to thank to Huynh Van Ngai for the fruitful discussion concerning the computation of the outer subdifferential in Example 6.2, and also to anonymous referees for their valuable comments. The second author was partially supported by the Research Plan MSM 4977751301.

\section{References}

1. Adly, S., Outrata, J.V.: Qualitative stability of a class of non-monotone variational inclusions. Application in electronics. J. Convex Anal. 20, 43-66 (2013)

2. Mordukhovich, B.S.: Variational Analysis and Generalized Differentiation. I. Basic Theory. Springer, Berlin (2006)

3. Dontchev, A.L., Rockafellar, R.T.: Implicit Functions and Solution Mappings. A View from Variational Analysis. Springer, Dordrecht (2009)

4. Mordukhovich, B.S., Wang, B.: Restrictive metric regularity and generalized differential calculus in Banach spaces. Int. J. Math. Math. Sci. 2004, 2653-2680 (2004)

5. Rockafellar, R.T., Wets, R.J.-B.: Variational Analysis. Springer, Berlin (1998)

6. Dontchev, A.L., Veliov, V.M.: Metric regularity under approximations. Control Cybern. 38, 1283-1303 (2009)

7. Henrion, R., Outrata, J.V.: Calmness of constraint systems with applications. Math. Program., Ser. B 104, 437-464 (2005)

8. Ioffe, A.D., Outrata, J.V.: On metric and calmness qualification conditions in subdifferential calculus. Set-Valued Anal. 16, 199-227 (2008)

9. Hoheisel, T., Kanzow, C., Outrata, J.V.: Exact penalty results for mathematical programs with vanishing constraints. Nonlinear Anal. 72, 2514-2526 (2010) 